Supporting Information

\title{
MAINMASTseg: Automated Map Segmentation Method for Cryo-EM Density Maps with Symmetry
}

Genki Terashi ${ }^{a}$, Yuki Kagaya ${ }^{b}$, and Daisuke Kihara ${ }^{a, c, *}$

${ }^{a}$ Department of Biological Sciences, Purdue University, West Lafayette, Indiana, 47907, USA

${ }^{\mathrm{b}}$ Graduate School of Information Sciences, Tohoku University, Aramaki-Aza, Aoba 6-3-09, Aoba-Ku Sendai, Miyagi, 980-8579, Japan

${ }^{\mathrm{c}}$ Department of Computer Science, Purdue University, West Lafayette, Indiana, 47907, USA

*Corresponding Author:

Daisuke Kihara: dkihara@purdue.edu 

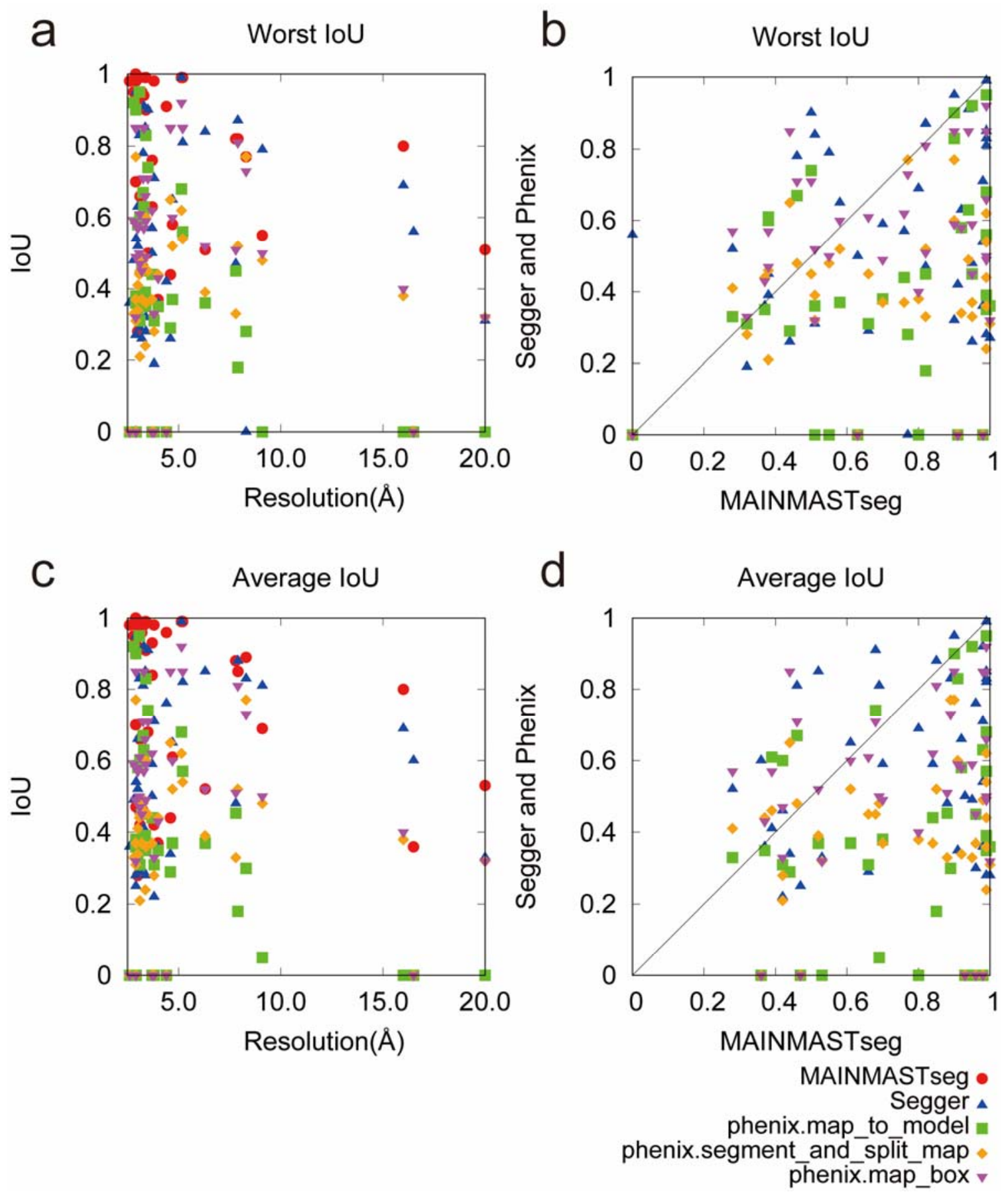

Figure S1. Comparison of MAINMASTseg, Segger, and three Phenix tools. (a) The worst-IoU values among chains in the reference structure in each map relative to the map resolution. (b) The worst-IoU values of Segger and Phenix tools against MAINMASTseg. (c) The average-IoU 
values of all chains in the reference structure relative to the map resolution. (d) The average-IoU values of Segger and Phenix tools against MAINMASTseg. Red circles, MAINMASTseg; blue triangles, Segger; green squares phenix.map_to_model; orange diamonds, phenix.segment_and_split_map; purple triangles, phenix.map_box. The plots for the best IoUs are shown in Figure 3 in the main text. 

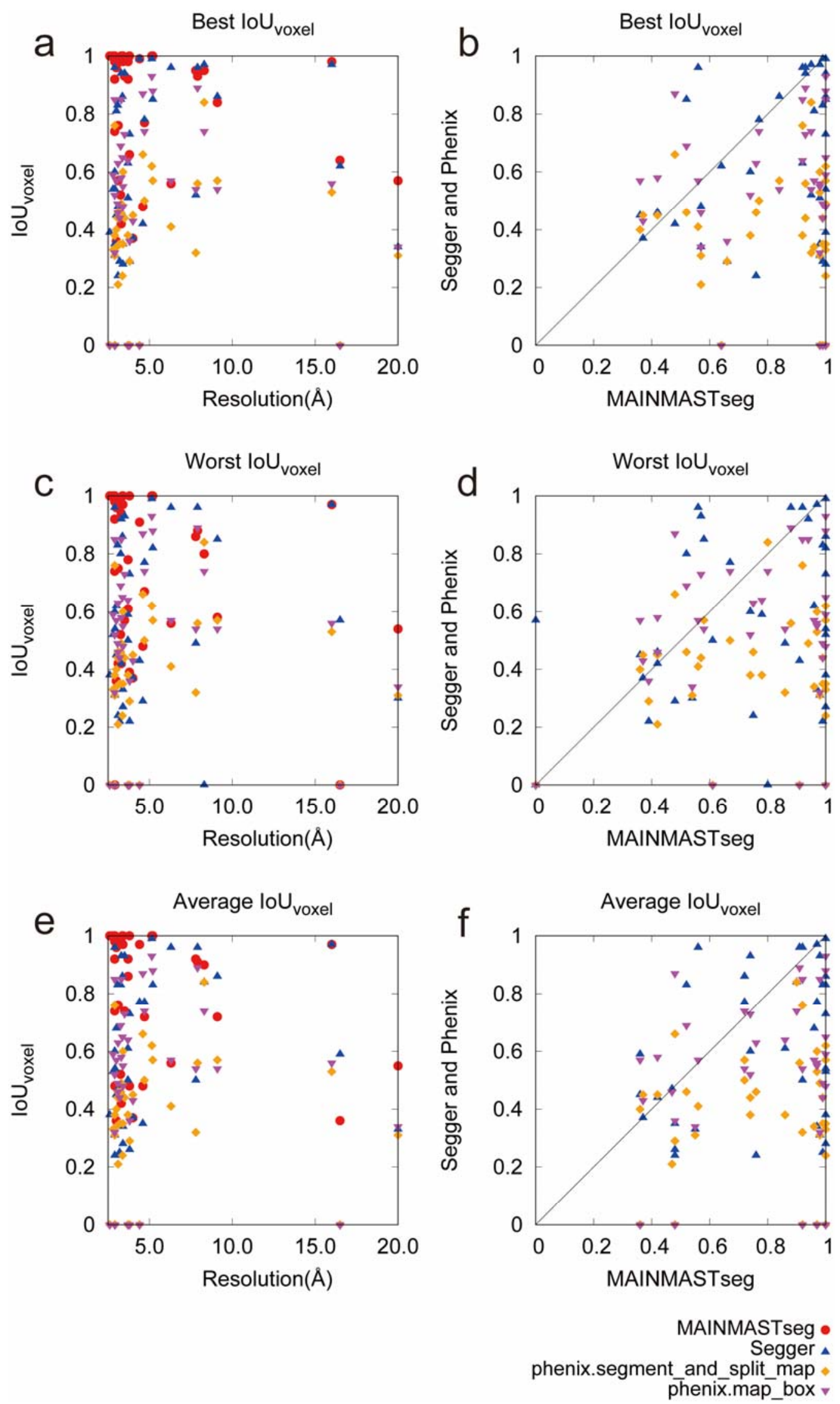
Figure S2. Comparison of MAINMASTseg with Segger, phenix.map_box and phenix.segment_and_split_map using IoUvoxel for evaluation. (a) The best IoUvoxel values relative to the map resolution. (b) Comparison of best $I_{\text {voxel }}$ values. (c) Worst- IoU $_{\text {voxel }}$ values relative to the map resolution. (d) Comparison of worst-IoU $U_{\text {voxel }}$ values. (e) Average-IoU voxel values relative to the map resolution. (f) Comparison of average-IoU $\mathrm{U}_{\text {voxel }}$ values.
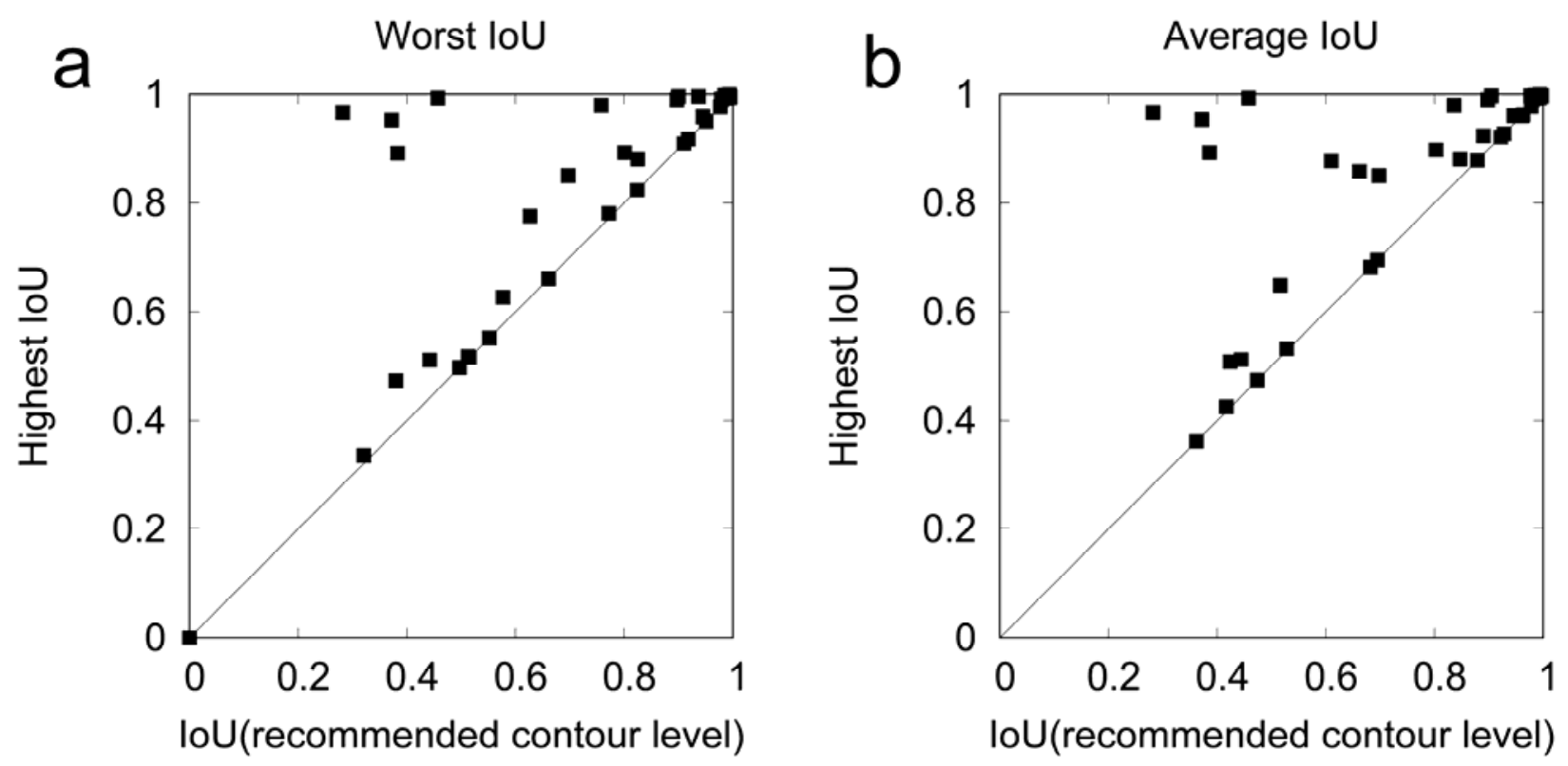

Figure S3. The worse and the average-IoU atom values of MAINMASTseg using three different contour levels. (a) The worst-IoUs. (b) The average-IoUs. The highest IoUs among three density contour levels (the recommended contour level*1.0, $0.75,0.5$ ) are plotted against the IoUs with the recommended contour level. The corresponding figures for the best IoUatom are provided in Figure 6 in the main text. 

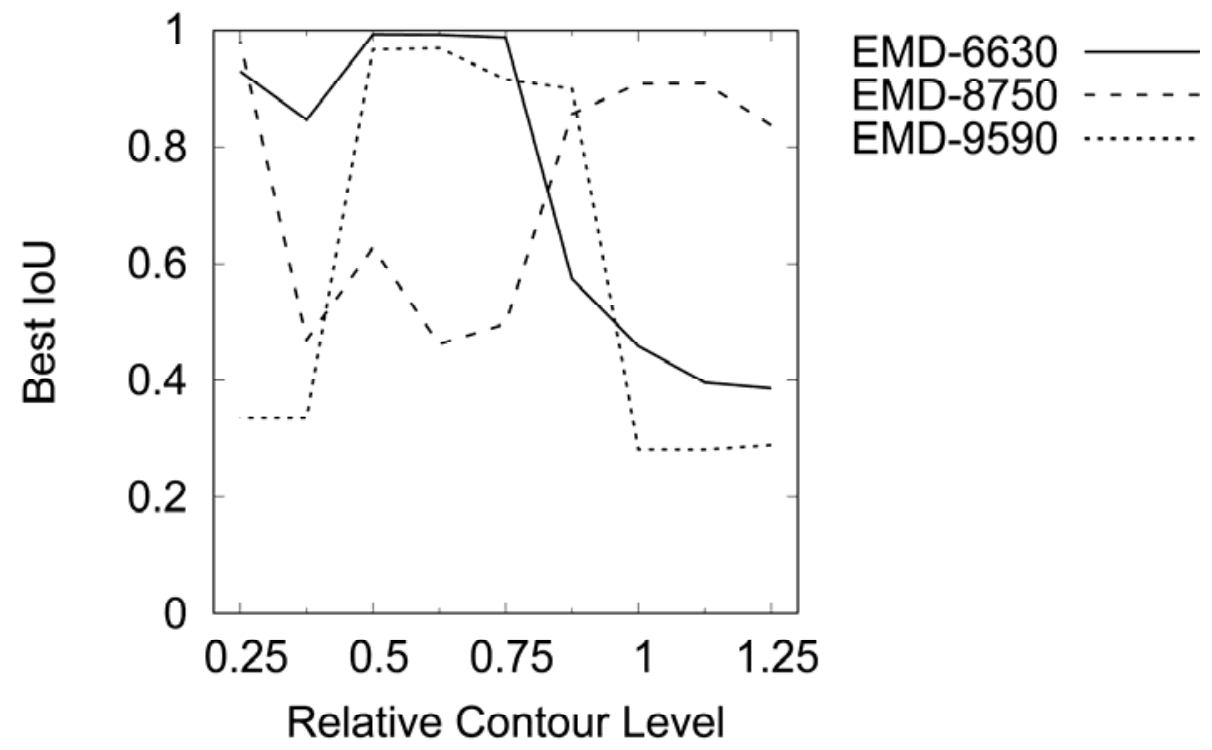

Figure S4. Best-IOUs of three EM maps using different contour levels. EMD-6630, EMD-8750, and EMD-9590 were used. Nine contour levels relative to the author-recommended levels were used: 1.25, 1.125, 1.0 (the author-recommended level), 0.875, 0.75, 0.625, 0.5, 0.375, and 0.25. 
Table S1. Segmentation accuracies of MAINMASTseg with different density thresholds

\begin{tabular}{|c|c|c|c|c|c|c|c|c|c|}
\hline \multirow[b]{2}{*}{ ID } & \multicolumn{3}{|c|}{ 1.0*author-level } & \multicolumn{3}{|c|}{ 0.75*author-level } & \multicolumn{3}{|c|}{ 0.5*author-level } \\
\hline & Best $^{\mathrm{a}}$ & Worst $^{\mathrm{b}}$ & $\mathrm{Ave}^{\mathrm{c}}$ & Best $^{\mathrm{a}}$ & Worst $^{\mathrm{b}}$ & $\mathrm{Ave}^{\mathrm{c}}$ & Best $^{\mathrm{a}}$ & Worst $^{\mathrm{b}}$ & Ave $^{\mathrm{c}}$ \\
\hline 0093 & 0.99 & 0.99 & 0.99 & 1.00 & 1.00 & 1.00 & 0.37 & 0.37 & 0.37 \\
\hline 0199 & 0.66 & 0.66 & 0.66 & 0.97 & 0.63 & 0.86 & 0.98 & 0.53 & 0.72 \\
\hline 0252 & 0.98 & 0.91 & 0.96 & 0.98 & 0.69 & 0.96 & 0.98 & 0.43 & 0.94 \\
\hline 0406 & 0.90 & 0.90 & 0.90 & 0.99 & 0.99 & 0.99 & 0.58 & 0.58 & 0.58 \\
\hline 0593 & 0.70 & 0.70 & 0.70 & 0.85 & 0.85 & 0.85 & 0.93 & 0.35 & 0.53 \\
\hline 0690 & 0.39 & 0.38 & 0.39 & 0.89 & 0.89 & 0.89 & 0.78 & 0.42 & 0.60 \\
\hline $1149^{d}$ & 0.64 & 0.00 & 0.36 & 0.64 & 0.00 & 0.36 & 0.64 & 0.00 & 0.36 \\
\hline $1871^{d}$ & 0.95 & 0.77 & 0.89 & 0.96 & 0.78 & 0.92 & 0.97 & 0.29 & 0.68 \\
\hline $1940^{d}$ & 0.54 & 0.51 & 0.53 & 0.55 & 0.51 & 0.53 & 0.55 & 0.52 & 0.53 \\
\hline $2355^{e}$ & 0.80 & 0.80 & 0.80 & 0.89 & 0.88 & 0.88 & 0.90 & 0.89 & 0.90 \\
\hline 3435 & 0.90 & 0.82 & 0.88 & 0.92 & 0.81 & 0.87 & 0.93 & 0.77 & 0.86 \\
\hline 3999 & 0.50 & 0.38 & 0.42 & 0.64 & 0.47 & 0.51 & 0.68 & 0.30 & 0.47 \\
\hline 4748 & 0.99 & 0.99 & 0.99 & 1.00 & 1.00 & 1.00 & 1.00 & 1.00 & 1.00 \\
\hline 4789 & 0.97 & 0.95 & 0.96 & 0.93 & 0.58 & 0.80 & 0.99 & 0.36 & 0.79 \\
\hline 4919 & 1.00 & 1.00 & 1.00 & - & - & - & - & - & - \\
\hline $5392^{d}$ & 0.81 & 0.55 & 0.69 & 0.79 & 0.50 & 0.66 & 0.80 & 0.51 & 0.66 \\
\hline 6272 & 0.98 & 0.98 & 0.98 & 0.99 & 0.99 & 0.99 & 1.00 & 0.52 & 0.74 \\
\hline $6458^{d}$ & 0.65 & 0.58 & 0.61 & 0.92 & 0.62 & 0.81 & 0.98 & 0.63 & 0.88 \\
\hline 6630 & 0.46 & 0.46 & 0.46 & 0.99 & 0.99 & 0.99 & 0.99 & 0.99 & 0.99 \\
\hline 6805 & 0.60 & 0.32 & 0.42 & 0.59 & 0.34 & 0.43 & 0.39 & 0.27 & 0.33 \\
\hline 7006 & 1.00 & 0.94 & 0.98 & 1.00 & 1.00 & 1.00 & 1.00 & 0.96 & 0.99 \\
\hline 7009 & 0.89 & 0.76 & 0.84 & 0.92 & 0.88 & 0.90 & 0.98 & 0.98 & 0.98 \\
\hline 7637 & 0.98 & 0.98 & 0.98 & 0.43 & 0.35 & 0.37 & 0.73 & 0.69 & 0.71 \\
\hline
\end{tabular}




\begin{tabular}{cccccccccc}
8072 & $\mathbf{0 . 9 9}$ & $\mathbf{0 . 9 9}$ & $\mathbf{0 . 9 9}$ & 0.56 & 0.33 & 0.45 & 0.84 & 0.84 & 0.84 \\
$8166^{f}$ & 0.52 & $\mathbf{0 . 5 1}$ & 0.52 & 0.48 & 0.45 & 0.47 & $\mathbf{0 . 7 1}$ & $\mathbf{0 . 5 1}$ & $\mathbf{0 . 6 5}$ \\
8438 & 0.99 & 0.99 & 0.99 & $\mathbf{1 . 0 0}$ & $\mathbf{1 . 0 0}$ & $\mathbf{1 . 0 0}$ & $\mathbf{1 . 0 0}$ & $\mathbf{1 . 0 0}$ & $\mathbf{1 . 0 0}$ \\
$8475^{f}$ & 0.87 & 0.82 & 0.85 & 0.87 & 0.87 & 0.87 & $\mathbf{0 . 8 8}$ & $\mathbf{0 . 8 8}$ & $\mathbf{0 . 8 8}$ \\
8750 & $\mathbf{0 . 9 1}$ & $\mathbf{0 . 5 0}$ & $\mathbf{0 . 6 8}$ & 0.50 & 0.44 & 0.48 & 0.63 & 0.45 & 0.48 \\
8946 & $\mathbf{0 . 9 9}$ & 0.63 & $\mathbf{0 . 9 3}$ & 0.93 & $\mathbf{0 . 7 8}$ & 0.90 & 0.94 & 0.59 & 0.83 \\
9116 & 0.91 & 0.90 & 0.91 & 0.99 & 0.99 & 0.99 & $\mathbf{1 . 0 0}$ & $\mathbf{1 . 0 0}$ & $\mathbf{1 . 0 0}$ \\
9212 & 0.95 & 0.95 & 0.95 & 0.96 & $\mathbf{0 . 9 6}$ & $\mathbf{0 . 9 6}$ & $\mathbf{0 . 9 8}$ & 0.65 & 0.93 \\
9325 & 0.93 & $\mathbf{0 . 9 2}$ & $\mathbf{0 . 9 2}$ & $\mathbf{0 . 9 7}$ & 0.74 & 0.90 & 0.96 & 0.62 & 0.80 \\
9590 & 0.28 & 0.28 & 0.28 & 0.92 & 0.91 & 0.92 & $\mathbf{0 . 9 7}$ & $\mathbf{0 . 9 7}$ & $\mathbf{0 . 9 7}$ \\
\hline 9705 & $\mathbf{0 . 9 6}$ & 0.00 & $\mathbf{0 . 4 7}$ & 0.76 & 0.00 & 0.41 & 0.72 & 0.00 & 0.37 \\
\hline 9798 & 0.98 & 0.98 & 0.98 & $\mathbf{0 . 9 9}$ & $\mathbf{0 . 9 9}$ & $\mathbf{0 . 9 9}$ & 0.65 & 0.48 & 0.49 \\
\hline 10188 & 0.45 & 0.44 & 0.44 & 0.45 & 0.45 & 0.45 & $\mathbf{0 . 5 1}$ & $\mathbf{0 . 5 1}$ & $\mathbf{0 . 5 1}$ \\
\hline 20028 & $\mathbf{0 . 9 9}$ & $\mathbf{0 . 9 9}$ & $\mathbf{0 . 9 9}$ & 0.33 & 0.33 & 0.33 & $\mathbf{0 . 9 9}$ & $\mathbf{0 . 9 9}$ & $\mathbf{0 . 9 9}$ \\
\hline 20265 & 0.37 & 0.37 & 0.37 & 0.33 & 0.33 & 0.33 & $\mathbf{0 . 9 5}$ & $\mathbf{0 . 9 5}$ & $\mathbf{0 . 9 5}$ \\
\hline Average & 0.80 & 0.70 & 0.75 & 0.81 & 0.68 & 0.76 & 0.83 & 0.62 & 0.74 \\
\hline
\end{tabular}

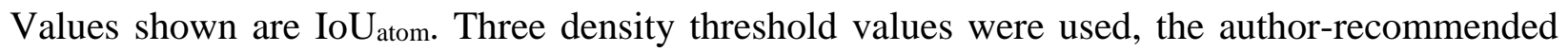
contour level, 0.75 of the author-recommended level, and 0.5 of the author-recommended level. In EMD-4919, MAINMASTseg could not generate segmented density maps when the contourlevel was lowered due to an increase of the map size that exceeded the memory size of the computer used. (a) the best IoU, (b) worst-IoU, (c) average-IoU, among all chains in the reference protein structure. The values in bold are the highest value for each map. (d) Using the band width of the gaussian filter $\boldsymbol{\sigma}=2.0 \AA$ (e) Using the band width of the gaussian filter $\boldsymbol{\sigma}=4.0$ $\AA$ (f) Using the band width of the gaussian filter $\boldsymbol{\sigma}=1.5 \AA$. 